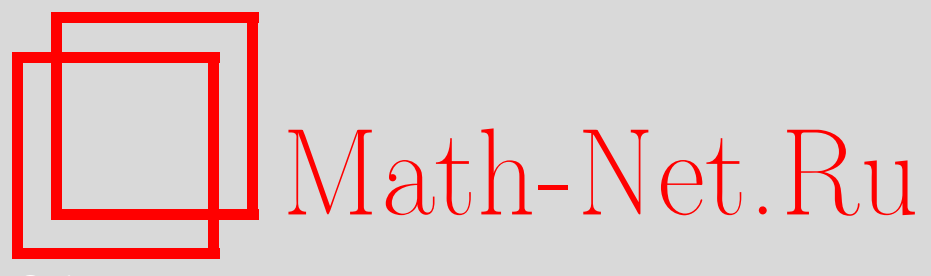

А. Я. Белов, А. Л. Чернятьев, Слова медленного роста и перекладывания отрезков, УМH, 2008, том 63, выпуск 1, 159-160

DOI: https://doi.org/10.4213/rm9128

Использование Общероссийского математического портала Math-Net.Ru подразумевает, что вы прочитали и согласны с пользовательским соглашением http://www . mathnet.ru/rus/agreement

Параметры загрузки:

IP: 54.84 .234 .179

26 апреля 2023 г., $14: 30: 25$

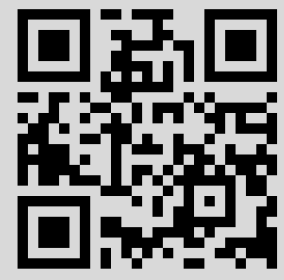




\title{
Слова медленного роста и перекладывания отрезков
}

\author{
А. Я. Белов, А. Л. Чернятьев
}

Пусть $W$ - сверхслово над конечным алфавитом $A$. Символ $x \in A-$ левое (правое) pacuирение $v$, если $x v$ (соответственно $v x$ ) есть подслово $W$. Подслово $v$ называется левым (правым) специальным подсловом, если для него существуют два или более левых (правых) расширения, и биспециалъным, если оно и левое, и правое специальное. Количество различных левых (правых) расширений подслова $v$ назовем левой (правой) валентностью в слове $W . k$-граф Рози слова $W$ - это такой граф: его вершины отвечают подсловам длины $k$ слова $W$, из вершины $A$ в вершину $B$ ведет стрелка, если в $W$ есть подслово длины $k+1$, у которого первые $k$ символов - подслово, соответствующее $A$, а последние $k$ символов - подслово, соответствующее $B$. Правым (левым) специальным словам соответствуют развилки вершины, из которых выходит (в которые входит) больше одной стрелки. Последователем графа Рози $G$ называется граф $D(G)$ : его вершины отвечают ребрам $G$, из вершины $A$ в вершину $B$ ведет стрелка, если в $G$ конечная вершина ребра $A$ является начальной вершиной ребра $B$. Последовательность $G_{1}, G_{2}, \ldots$ назовем эволюиией графов Рози данного слова $W$. При переходе от $G_{n}$ к $G_{n+1}$ возможны следующие варианты.

1. В графе $G_{n}$ нет сцепленных циклов (т. е. нет входящих развилок, являющихся одновременно исходящими). В этом случае граф $G_{n+1}$ совпадает с последователем $D\left(G_{n}\right)$.

2. В графе $G_{n}$ одна развилка является одновременно входящей и выходящей. Граф последователя $D\left(G_{n}\right)$ в этом случае имеет три развилки, так как одна развилка размножилась. Следовательно, граф $G_{n+1}$ получается из последователя $D\left(G_{n}\right)$ путем удаления одного ребра, соответствующего минимальному невстречающемуся слову.

3. В графе есть не менее двух развилок, являющихся одновременно входящими и выходящими. Граф $G_{n+1}$ получается из $D\left(G_{n}\right)$ удалением двух или более ребер, соответствующих минимальным невстречающимся словам.

С отображением $f: M \rightarrow M$ компакта в себя, его разбиением на $U_{1}, \ldots, U_{n}$ и начальной точкой $x_{0}$ связана символическая динамика (см. [1]).

Если точка принадлежит множеству $T^{-n}\left(U_{i_{n}}\right) \cap T^{-(n-1)}\left(U_{i_{n-1}}\right) \cap \cdots \cap T^{-1}\left(U_{i_{1}}\right) \cap U_{i_{0}}$, то эволюция начинается со слова $a_{i_{0}} a_{i_{1}} \cdots a_{i_{n}} . I_{u}$ - множество разбиений, которое соответствует слову $u$. Для слова $u$ назовем слово $v$ левым (правым) потомком, если $u$ - суффикс (префикс) слова $v$, в соответствии с этим будем называть вершину в $G_{n}$ левым (правым) потомком вершины в $G_{k}, n>k$. Прообраз конца интервала может являться граничной точкой только для двух интервалов; соответственно, специальные подслова могут иметь валентность только равную 2.

Правило 1. Чтобы слово $W$ порождалось перекладыванием отрезков, необходимо, чтобы при $k \geqslant k_{0}$ все $k$-графы Рози имели развилки только степени 2.

Пусть некоторому подслову $w$ соответствует характеристический интервал, лежащий внутри интервала перекладывания, точка $A \in[0,1]$ делит $I_{w}$ на два интервала, образы которых лежат в $I_{a_{k}}$ и $I_{a_{l}}$ соответственно, а точка $B \in[0,1]$ - делит $I_{w}$ на интервалы, прообразы которых лежат в $I_{a_{i}}$ и $I_{a_{j}}$ соответственно. Выбор минимального невстречающегося слова (т. е. удаляемого ребра) определяется взаиморасположением точек $A$ и $B$, сохранением или сменой ориентации отображения на характеристических множествах, всего 8 вариантов.

Граф Рози $G_{1}-$ размеченный, если: 1) ребра каждой развилки помечены символами $l$ ("left") и $r$ ("right"); 2) некоторые вершины помечены символом "-". Последователем $G_{1}$ назовем граф, являющийся его последователем как графа Рози, такой, что:

Работа выполнена при поддержке Israel Science Foundation (грант № 1178/06). 
1) ребра, входящие в развилку, помечены теми же символами, что и ребра, входящие в любого левого потомка этой вершины; 2) ребра, выходящие из развилки, помечены теми же символами, что и ребра, выходящие из любого правого потомка этой вершины; 3) если вершина помечена знаком "-", то все ее правые потомки помечены знаком "-".

ЗАмечАниЕ. Пусть ребра входящей развилки соответствуют $a_{i}$ и $a_{j}$, символы $l$ и $r$ соответствуют левому и правому множеству в паре $\left(T\left(I_{a_{i}}\right), T\left(I_{a_{j}}\right)\right)$. Если символы $a_{k}$ и $a_{l}$ соответствуют ребрам исходящей развилки, то символы $l$ и $r$ ставятся в соответствии с порядком “лево-право" в паре $\left(I_{a_{k}}, I_{a_{m}}\right)$. Знак “-” ставится в вершине, если характеристическое множество, ей соответствующее, принадлежит интервалу перекладывания, на котором меняется ориентация.

Условие для перехода от графа $G_{n}$ к $G_{n+1}$ :

ПрАвило 2. 1) Если в графе нет двойных развилок, соответствующих биспециальным подсловам, то при переходе от $G_{n}$ к $G_{n+1}$ имеем $G_{n+1}=D\left(G_{n}\right)$.

2) Если вершина помечена знаком “-”, то удаляемые ребра должны выбираться из пары $l l$ или $r r$.

3) В противном случае ребра, соответствующие запрещенным словам, выбираются из пар $l r$ и $r l$.

Эволюция размеченных графов Рози правильна, если правила 1 и 2 выполняются для всей цепочки эволюции графов, начиная с $G_{1}$, и асимптотически правильна, если правила 1 и 2 выполняются, начиная с некоторого $G_{n}$. Эволюция размеченных графов Рози ориентирована, если в $k$-графах нет вершин, помеченных знаком “-".

Теорема. Равномерно-рекуррентное слово $W$

1) порождается перекладыванием отрезков тогда и толъко тогда, когда $W$ обеспечивается асимптотически правильной эволючией размеченных графов Рози;

2) порождается перекладыванием отрезков с сохранением ориентации тогда и только тогда, когда $W$ обеспечивается асимптотически правильной ориентированной эволюиией размеченных графов Рози.

НАБРОСОК ДОКАЗАТЕЛЬСТВА. Пусть эволюция устроена хорошим образом. На первом этапе построим кусочно непрерывное преобразование, которое будет порождать данное слово. Разобьем отрезок на достаточно большое количество интервалов, которые соответствуют буквам алфавита, и будем поэтапно определять образы концевых точек согласованно с эволюцией графов. Далее продолжаем отображение по непрерывности (отметим, что условие согласованности при выборе запрещенного ребра в графах соответствует тому, что отображение имеет конечное число точек разрыва). Имеется инвариантная мера. Рассмотрим топологию, склеивающую точки с нулевым расстоянием, и построим соответствующую фактординамику. Полученное отображение и будет перекладыванием отрезков.

\section{Список литературы}

[1] А.Я. Белов, Г.В. Кондаков, Фундам. и прикл. матем., 1:1 (1995), 71-79. [2] M. Lothaire, Algebraic combinatorics on words, Encyclopedia Math. Appl., 90, Cambridge Univ. Press, Cambridge, 2002.

\section{А. Я. Белов (А. Yа. Belov)}

Московский институт открытого образования International University Bremen, Germany

E-mail: akanel@mail.ru, kanel@mccme.ru
Представлено А. В. Михалёвым Принято редколлегией 15.07.2007

\section{А. Л. Чернятьев (A. L. Chernyatiev)}

Московский государственный университет

им. М. В. Ломоносова

E-mail: chernyatiev@gmail.com 